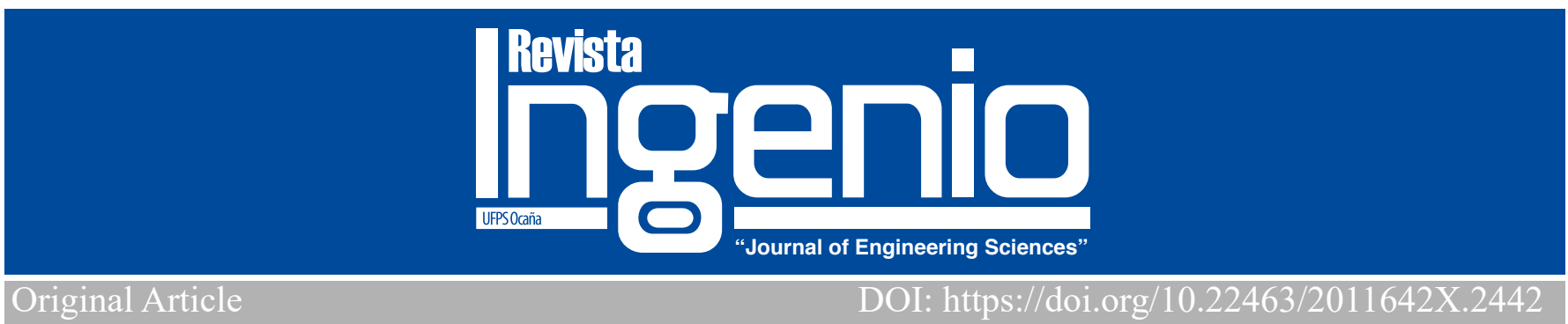

\title{
Automatización de un sistema de extracción, llenado y distribución de agua potable en Ocaña, Colombia
}

\author{
Automation of a drinking water extraction, filling and distribution system in Ocaña, Colombia \\ MSc. Eduar Pérez Rojas ${ }^{1}$, Esp. Camilo Lobo Guerrero², Ing. Duvan Sánchez Quintana ${ }^{3}$
}

${ }^{1}$ Estándar Ingenieria SAS. Aguachica, Colombia, Orcid: https://orcid.org/0000-0003-2971-3453; https: //orcid.org/0000-0003-0460-658X, Email:ing.eduar10@hotmail.com
2 SERINGTEC. Bogotá, Colombia, Orcid: https://orcid.org/0000-0002-4333-9958, Email: camilo.lobo@seringtec.com
${ }_{3}^{3}$ Estándar Ingenieria SAS. Aguachica, Colombia, Orcid: https://orcid.org/0000-0003-2971-3453; https://orcid.org/0000-0003-0460-658X, Email:dusanquin@hotmail.com

Como citar: E. Pérez-Rojas, C. Lobo-Guerrero \& D. Sánchez- Quintana, “Automatización de un sistema de extracción, llenado y distribución de agua potable en Ocaña, Colombia”, Revista Ingenio, vol. 15 (1), pp. 1-7, 2018.

Fecha de recibido: 02 de junio de 2017 Fecha de aprobado: 25 de octubre de 2017

\section{RESUMEN}

Palabras claves:

Agua, Acueducto, Comunicación, Control, Optimización.

\begin{abstract}
En la presente investigación se llevó a cabo el diseño de una propuesta del sistema de automatización para la extracción, llenado y distribución de agua potable en el municipio de Ocaña, Colombia. Inicialmente, se determinó la ubicación geográfica de la planta de tratamiento de agua potable del algodonal, tanques de distribución y el punto geográfico más alto del municipio, con la finalidad de establecer la estrategia de comunicación y control de las etapas de llenado y distribución de agua en los tanques. Los resultados demuestran que el corregimiento de Pueblo Nuevo es el punto geográfico estratégico donde se debe ubicar la antena repetidora para evitar interferencias de señal del control de los tanques. Finalmente, gracias a las estrategias de control y comunicación diseñadas, se optimiza la pérdida de agua relacionada con daños externos en el sistema de acueducto y por lo tanto beneficia económicamente a la empresa del municipio.
\end{abstract}

\section{ABSTRACT}

Key words: In the present work, the design of a proposal of the automation system for the extraction, filling, and distribution of drinking water
was carried out in the municipality of Ocaña, Colombia. Initially, the geographical location of the Algodonal drinking water treatment
plant, distribution tanks, and the highest geographical point of the municipality were determined to establish the communication and
control strategy for the stages of filling and distribution of water in the tanks. The results show that the township of Pueblo Nuevo
is the strategic geographical point where the repeater antenna should be located to avoid signal interference from the tank control.
Finally, thanks to the control and communication strategies designed, the loss of water-related to external damages in the aqueduct
system is optimized and, therefore, economically benefiting the municipality's company.

\section{Introducción}

Actualmente en Colombia, el sistema de acueducto de las empresas públicas de Medellín E.S.P. [1], está compuesto por subsistemas interconectados entre sí, controlados mediante un Sistema de Supervisión y Adquisición de Datos (SCADA) con el cual se garantiza el abastecimiento de cada uno de los puntos geográficos de la ciudad y por lo tanto la optimización del fluido (agua).

Por otra parte, los grandes productores de sistemas de automatización industrial ofrecen paquetes para el control total de las plantas de agua potable como es el caso de S. Electric [2], los cuales diseñaron un procedimiento de soluciones para el sector del agua potable y aguas residuales, ofreciendo una amplia gama de soluciones para este sector. Asimismo, Unatsabar [3] desarrolló guías/procedimientos para el diseño de estaciones de bombeo de agua potable; en este documento se presentan los parámetros y criterios para una correcta distribución de agua potable en poblaciones menores a 2000 habitantes.

El sistema SCADA, es un concepto que se utiliza para controlar procesos industriales a distancia, facilitando la supervisión en tiempo real con dispositivos electrónicos (sensores y actuadores) desde un lugar remoto desde donde se puede visualizar, controlar y almacenar datos de las variables que se están monitoreando. La Figura 1, muestra un sistema SCADA compuesto de una MTU (Unidad Terminal Maestra), de un RTU (Unidades Terminales Remotas) y los medios físicos de comunicación, siendo el diseño básico de operación para este tipo de sistemas. 


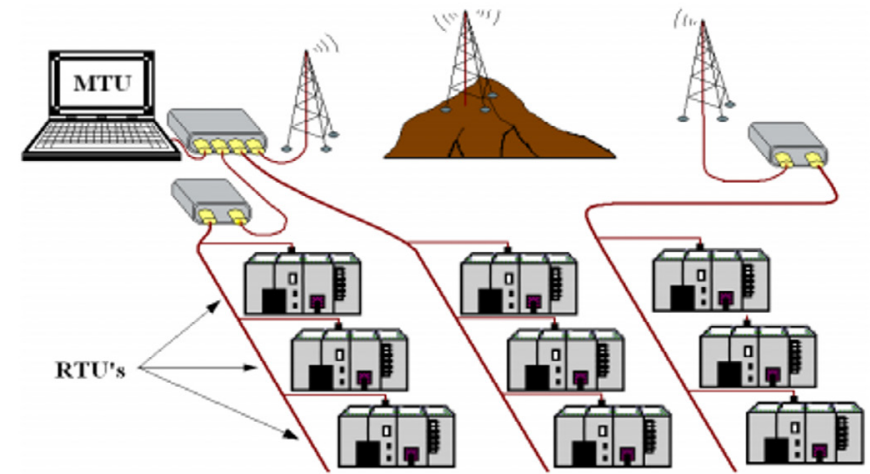

Figura 1. Sistema SCADA. Fuente. [4-5]

El propósito general de este trabajo es evitar que el control del sistema de llenado de los tanques y activación de la bomba dependa de la comunicación entre los operarios y sea a través de señal digital que permita un mayor control y optimización del proceso. Además, se propone diseñar el sistema de control automático que permite manipular las variables del sistema en tiempo real con un sistema SCADA, mediante software libre e interactivo para que el operario pueda utilizarla fácilmente.

\section{Materiales y métodos}

El acueducto se encuentra localizado en Ocaña, al nororiente colombiano. El punto inicial tiene lugar en la bocatoma del río Algodonal con coordenadas: N8¹3'10.81', W73 $19^{\prime} 14.20 ”$ ', y está a 1204 m.s.n.m. Este se encuentra ubicado aproximadamente a $5 \mathrm{~km}$ del centro de la ciudad. Desde este punto se bombea aguas hasta el tanque Buenavista que tiene coordenadas: N8 $8^{\circ} 11^{\prime} 18.42^{\prime \prime}, \mathrm{W} 73^{\circ} 20^{\prime} 45.53^{\prime \prime}$ y está a 1660 m.s.n.m. Este tanque encuentra a $4 \mathrm{~km}$ de la bocatoma y este tanque cuenta con la cota más alta dentro del sistema de acueducto. Posteriormente se distribuye mediante gravedad a los tanques "el llanito" con coordenadas N 8'13'25.73”, W 7321'19.10” y está a 1358 m.s.n.m. que se encuentra a $4 \mathrm{Km}$ y tiene del tanque Buenavista y "Cristo Rey" con coordenadas N 8०14'26.47”, W 73²0'59.65” y está a 1262 m.s.n.m. que se encuentra a $5.7 \mathrm{~km}$ y tiene del tanque Buenavista como se observa en la Figura 2.

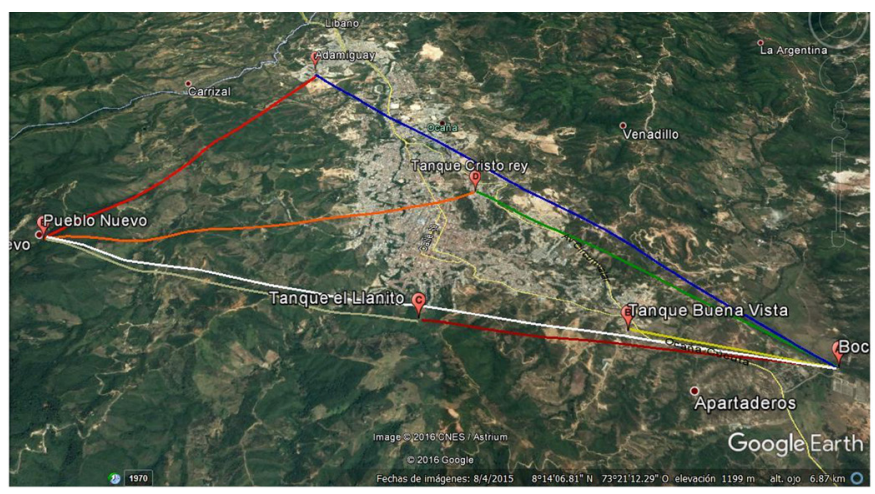

Figura 2. Ubicación geográfica de los tanques y posibles soluciones de comunicación.
El control de un proceso es fundamental para cualquier empresa, mediante el cual se pueden manipular las variables de un sistema y de esta manera adecuarlas a requerimientos particulares. Para este caso, se llevó a cabo la automatización del sistema de extracción, llenado y distribución de agua para el municipio de Ocaña Norte de Santander, Colombia. El desarrollo de esta propuesta se basa en el suministro de agua, teniendo en cuenta que esta empresa toma el líquido-fluido (agua) del río Algodonal mediante una bomba ubicada en el batallón Santander, este equipo es el encargado de llenar unos tanques ubicados en puntos estratégicos para la empresa. El proceso de llenado de los tanques empieza cuando el sistema de bombeo es activado, y distribuye el agua a los tanques ubicados en la entrada del sector de Buenavista y posteriormente al sector del Llanito y Simón Bolívar (tanques principales).Posteriormente estos suministran el fluido a tanques secundarios ubicados en puntos estratégicos para la empresa. Cuando los tanques han alcanzado el máximo nivel permitido, uno de los operarios se comunica con el operador de la bomba para que la apague o para que cierre la válvula que le suministra a los mismos.

\subsection{Diseño del sistema.}

El diseño del sistema de automatización para el método de extracción, llenado y distribución de agua de la empresa permite el control de las variables que intervienen en el sistema mismo, con el cual se disminuyen las pérdidas generadas en los procesos de llenado y distribución de agua potable en el municipio. Actualmente la empresa servicios públicos de Ocaña cuenta con una capacidad de bombeo máxima. Para garantizar el abastecimiento completo del sistema de acueducto del municipio de Ocaña, desde la bocatoma del río Algodonal se bombean 30 lt/min, hasta el tanque Buenavista. Desde este tanque se redirecciona o rebombea a los tanques del Llanito y Cristo Rey con una tasa de flujo de $11 \mathrm{lt} / \mathrm{min}$, de acuerdo con el Plan Maestro Acueducto y Alcantarillado Ocaña [4]. La capacidad de cada uno de los tanques se muestra en la Tabla 1. 
Tabla 1. Capacidad del tanque de Buenavista.

\begin{tabular}{llll}
\hline Tanque & Capacidad $\left(\mathrm{m}^{3}\right)$ & Sección superficial $(\mathrm{m})$ & Profundidad $(\mathrm{m})$ \\
\hline Buenavista & 2000 & $23.97 * 23.93$ & 3.5 \\
\hline Cristo Rey & 3000 & $30.05^{*} 29.95$ & 3.4 \\
\hline El llanito & 1900 & $40.70 * 12.70$ & 3.6 \\
\hline
\end{tabular}

Fuente: [6]

El tanque Buenavista, actualmente cuenta con un sistema de control manual donde se ubica un operario que realiza una inspección visual del llenado, generando gasto de tiempo productivo en horas hombre.

\section{Resultados y discusiones}

3.1. Comparativo de controladores.

Un controlador automático compara el valor real de la salida de una planta con la entrada de referencia (el valor deseado), determina la desviación y produce una señal de control que reduce la desviación a cero o a un valor pequeño. La manera en la cual el controlador automático produce la señal de control se denomina acción de control [7-8-9-10].

\subsection{Captación de agua del río Algodonal.}

El proceso inicia con la captación de agua del río Algodonal por medio de la bocatoma lateral, esta permite el paso de agua hacia la piscina desarenador (PIS-001) por medio de la válvula motorizada (MOV-001). El nivel de llenado por alto, se controla con el interruptor de nivel SLH-001, cerrando la válvula MOV-001 cuando el interruptor SLH-001 envía la señal (24 Vdc) al PLC-001. Para desactivar el interruptor SLH-001 cuando el nivel en la piscina es bajo, se envía una señal (0 Vdc), que permite la apertura de la válvula MOV-001 nuevamente. Las unidades de bombeo P-001 / P-002 / P-003 / P-004 y P-005 realizan el traslado de agua desde la piscina desarenador PIS-001 a la piscina de tratamiento floculador (PIS-002); las unidades de bombeo pueden estar en estado de marcha (start) o detenido (stop) de forma local desde el tablero de control de cada motor o de forma remota desde el PLC-001 siempre y cuando el selector Local/Remoto esté en su respectiva posición. La parada o encendido de las bombas tiene un interlock (permisivo) de control con el interruptor por bajo nivel (SLL-001) de la piscina desarenador con el fin de que las unidades de bombeo no estén encendidas cuando la piscina PIS-001 se encuentre con bajo nivel, esto para protección de la integridad de las bombas (Tabla 2). De igual forma, las unidades de bombeo P-001 / P-002 / P-003 / P-004 y P-005 tienen otro interlock (permisivo) con los interruptores por alto nivel (SLH-002/SLH-003/SLH-004) de la piscina de tratamiento PIS-002 apagando las bombas para evitar sobrellenado de la piscina de tratamiento PIS002 .

Tabla 2. Comparativo de controladores

\begin{tabular}{|c|c|c|c|c|}
\hline & $\mathrm{N}$ & Detalle & Micro820 & Micro870 \\
\hline & 1 & $\begin{array}{l}\text { Puertos de } \\
\text { comunicación } \\
\text { incorporados }\end{array}$ & $\begin{array}{l}\text { - Puerto 10/100 Base } \\
\text { T/Ethernet/ RJ-45 } \\
\text { - Composición de } \\
\text { RS232/RS485/en serie no } \\
\text { aislado }\end{array}$ & $\begin{array}{l}\cdot \text { EtherNet/IP Clase 3/TCP- } \\
10 / 100 \mathrm{Mbps} \\
\text { - RS232/485, Modbus RTU } \\
\text { maestro/esclavo, ASCII, CIP }\end{array}$ \\
\hline & 2 & $\begin{array}{l}\text { Puntos de } \\
\text { E/S/digitales }\end{array}$ & 19 & 24 \\
\hline & 3 & $\begin{array}{l}\text { Canales de E/S/ } \\
\text { analógicos }\end{array}$ & $\begin{array}{l}\text { - Salida analógica de } 0-10 \mathrm{~V} \\
\text { - Cuatro entradas digitales de } 24 \\
\text { VCC configuradas como } \\
\text { analógicas de } 0-10 \mathrm{~V} / \mathrm{módulos} \\
\text { enchufables }\end{array}$ & $\begin{array}{l}\text { Mediante módulos } \\
\text { enchufables o con módulos } \\
\text { E/S/expansión }\end{array}$ \\
\hline \multirow{4}{*}{ CARACTERÍSTICAS } & 4 & $\begin{array}{l}\text { Cantidad de } \\
\text { módulos } \\
\text { enchufables }\end{array}$ & 2 & 3 \\
\hline & \multirow{3}{*}{5} & $\begin{array}{l}\text { Cantidad de } \\
\text { módulos de } \\
\text { expansión }\end{array}$ & 0 & 8 \\
\hline & & $\begin{array}{l}\text { Máx. de } \\
\text { E/S/digitales }\end{array}$ & 35 & 304 \\
\hline & & Tipos de & $\begin{array}{l}\text { - Micro800/LCD remoto/2080- } \\
\text { REMLCD }\end{array}$ & $\begin{array}{l}\cdot \text { Micro800/LCD } \\
\text { remoto/2080-REMLCD }\end{array}$ \\
\hline
\end{tabular}


6 accesorios/módul os/enchufables

- Excepto 2080 MEMBAKRTC/ módulos enchufables

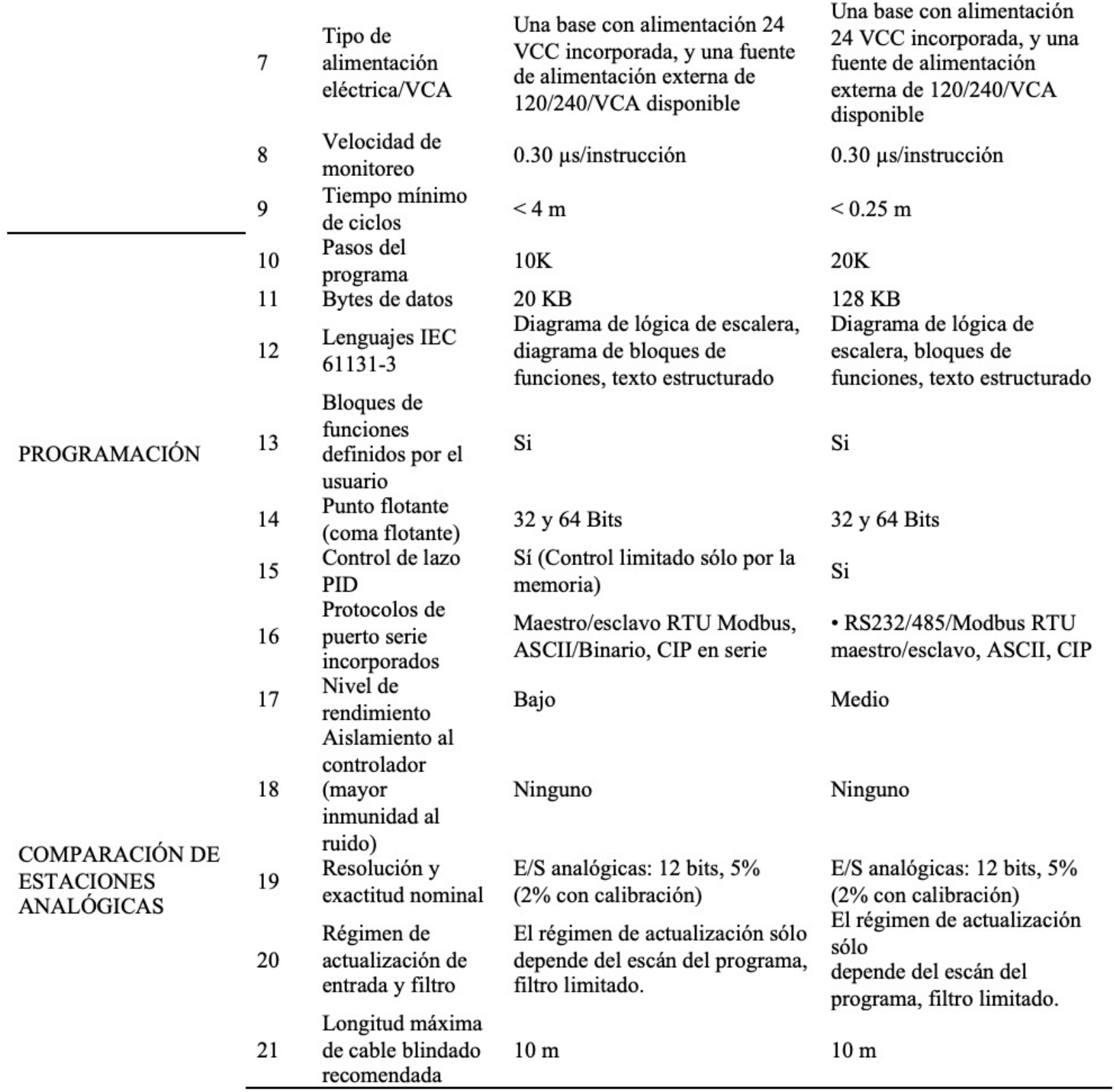

- Excepto 2080 MEMBAK$\mathrm{RTC} /$ módulos enchufables $24 \mathrm{VCC}$ incorporada, y una

$<0.25 \mathrm{~m}$

Diagrama de lógica de escalera, bloques de funciones, texto estructurado 
(Figura 3). Las unidades de bombeo pueden estar en estado de marcha (start) o detenido (stop) de forma local desde el tablero de control de cada motor o de forma remota desde el PLC-001 siempre y cuando el selector Local/Remoto esté en la respectiva posición deseada. La parada o encendido de las bombas tiene un interlock de control con los interruptores por bajo nivel (SLL-002/SLL-003/SLL-004) de cada una de las líneas de tratamiento de la piscina PIS-002 con el fin de que las unidades de bombeo no estén encendidas cuando la piscina PIS-002 se encuentre con bajo nivel para protección de la integridad de las bombas, de igual forma las unidades de bombeo P-006 / P-007 y P-008 tienen otro interlock con el interruptor por alto nivel (SLH-005) del tanque de almacenamiento de Buenavista enviando una señal vía radio frecuencia al PLC-001 para apagado de las bombas y cerrado de la válvula motorizada MOV-005, para que se pueden volver a disponer en marcha (start) una vez el interruptor por alto nivel (SLH-005) del tanque de almacenamiento de Buenavista se encuentre desactivado [11-13-14-15-16-17].

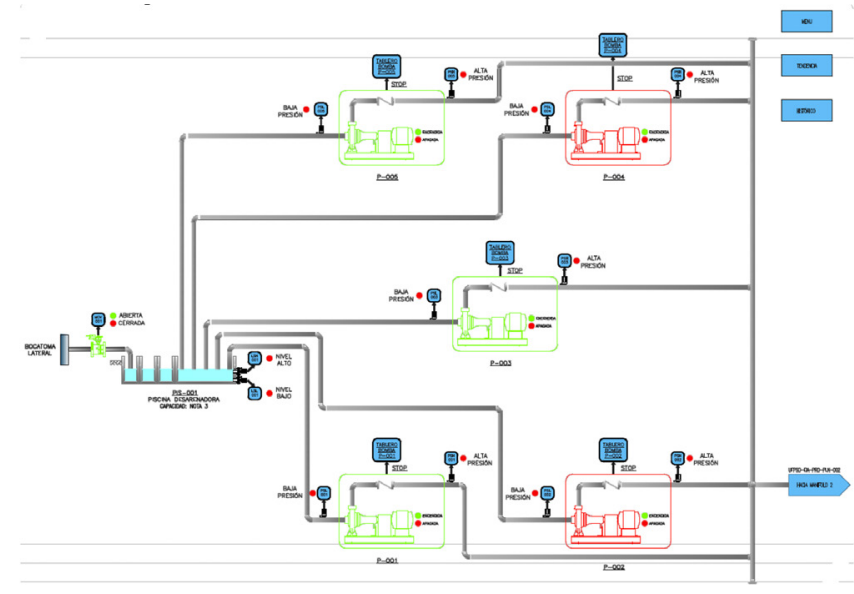

Figura 3. Esquema de la interfaz HMI del control de llenado de cada una de las líneas de tratamiento de agua

\subsection{Control de llenado del tanque de almacenamiento}

Buenavista (TK-001)

Si el interruptor por alto nivel (LSH-005) del tanque de Buenavista (TK-001) no se encuentra activo $(0 \mathrm{Vdc})$ las unidades de bombeo P-006 / P-007 y P-008 pueden estar en estado encendido (start) siempre y cuando el otro permisivo con los interruptores por bajo nivel de la PIS-002 se encuentre habilitado, con esas acciones la válvula motorizada MOV005 se encuentra en estado abierta y empieza el llenado del tanque de almacenamiento de Buenavista, tan pronto el nivel de agua en el tanque active el interruptor por alto nivel (LSH$005)$ envía la señal digital $(24 \mathrm{Vdc})$ a la RTU-001 y éste a su vez envía la señal vía radio frecuencia al controlador maestro ubicado en el Acueducto Algodonal PLC-001 para apagado de las unidades de bombeo P-006 / P-007 y P-008 y cierre de la válvula motorizada MOV-005 (Figura 4).

Las unidades de bombeo P-009 y P-010 realizan el transporte de agua desde el tanque de Buenavista TK-001 al tanque de almacenamiento auxiliar de Buenavista (TK-002), Llanito (TK- 003) y Cristo Rey (TK-004). Las unidades de bombeo pueden estar en estado de marcha (start) o detenido (stop) de forma local desde el tablero de control de cada motor o de forma remota desde el PLC-001 siempre y cuando el selector Local/Remoto esté en su respectiva posición. Las unidades de bombeo P-009 y P-010 tienen un interlock por alto nivel de los tanques TK-002, TK003 y TK-004, solo cuando los tres interruptores de alto nivel LSH-006, LSH007 y LSH-008 se encuentren activos (24Vdc) las bombas se apagan, mientras uno de los tres interruptores se encuentre desactivado $(0 \mathrm{Vdc})$ las bombas permanecerán encendidas.

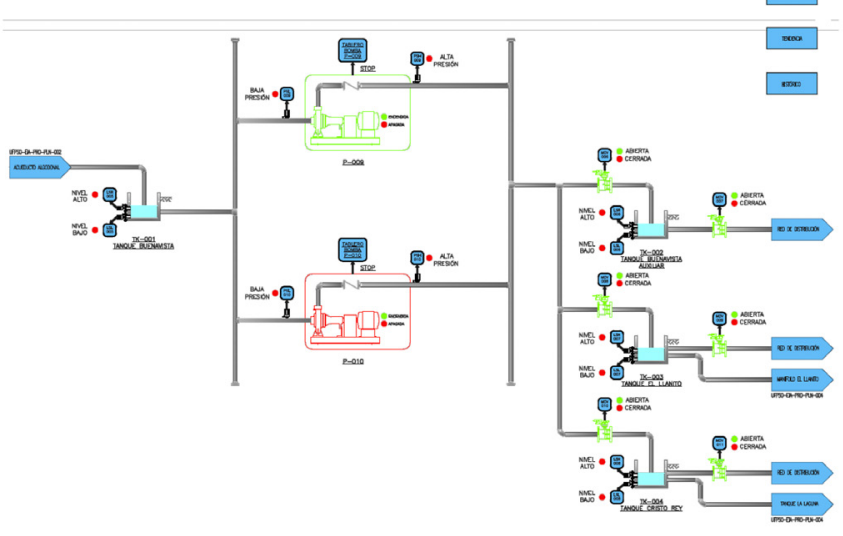

Figura 4. Esquema de la interfaz HMI del Control de llenado del tanque de almacenamiento Buenavista (TK-001).

3.5. Diseño del sistema de comunicación

Arquitectura de control, se presenta el diseño del sistema de comunicación donde se muestra el sistema de control que cuenta con el controlador lógico programable PLC-001 y 9 unidades terminales remotas RTU ubicadas en cada uno de los tanques (Figura 5a). Todas las señales asociadas a los equipos de los tanques de almacenamiento serán comunicadas vía radio frecuencia al PLC-001 utilizando las antenas repetidoras ubicadas en Pueblo Nuevo (Figura 5b). El cableado desde el radio modem hacia el SWITC de comunicaciones en cada RTU se realizará mediante cable ETHERNET, utilizando el protocolo MODBUS TCP/IP (Figura 5c). 


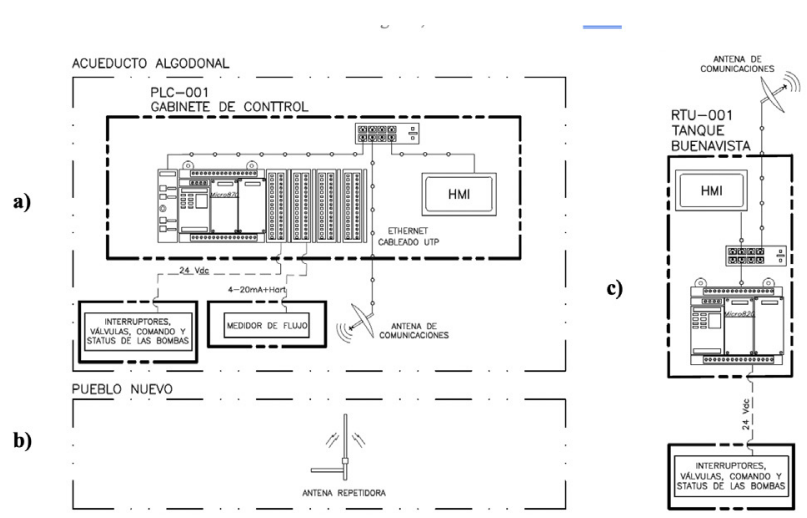

Figura 5. Sistema de comunicaciones. a) Gabinete de control, b) Antena repetidora y c) Sistema RTU.

\section{Conclusiones}

Para el sistema de transmisión de señales de procesos desde los tanques satélites a la planta de tratamiento de agua el "Algodonal" se realiza a nivel conceptual extendida el cual se requiere verificar distancia y línea de vista entre los puntos, se recomienda para el desarrollo de la ingeniería de detalle realizar el estudio de propagación el cual nos arrojen los resultados tales como pérdidas por obstrucción, urbanización e interferencias, latitud y longitud de las antenas, alturas de instalación para cálculos de la inclinación del lóbulo de la señal de propagación los cuales nos permitirá calcular la confiabilidad del sistema de comunicación y garantizar la respectiva optimización del dimensionamiento de las antenas que nos proporcione la eficiencia necesaria para transmitir las señales de procesos tales como niveles de llenado de los tanques para la supervisión remotamente e interlocks para encendido y apagado de las bombas.

El nivel de automatización y control del sistema del "sistema de extracción, llenado y distribución de agua para el municipio de Ocaña, tomado para el desarrollo del presente trabajo de investigación es de tipo on/off, dado que el sistema de proceso no es crítico; es decir, que no causa afectaciones en infraestructura y riesgo humano por manejar niveles de presiones bajos, y el almacenamiento en los tanques es a presiones atmosféricas. Finalmente, es recomendable la utilización de lógica inversa, la cual permitirá una seguridad y control del proceso sin tener instrumentación de respaldo.

\section{Referencias}

[1] Empresas públicas de Medellín. (2015) "Sistema de acueducto de las empresas públicas de Medellín E.S.P." [Online]. Available: https://www.epm. com.co/site/Portals/0/centro_de_documentos/ NormasDisenoSistemasAcueducto.pdf

[2] S. Electric, (2011) Guía de soluciones para el sector del agua potable y aguas residuales, América del sur:
Schneíder Electric, [Online].Available: https://www. se.com/es/es/work/solutions/for-business/water/

[3] Unatsabar, (2005) "Guías para el diseño de estaciones de bombeo de agua potable," Organización Panamericana de la Salud, Lima,[Online]. Available: https://sswm.info/sites/default/files/ reference_attachments/OPS $\% 202005 \mathrm{~b} \% 20 \mathrm{Guia} \% 20$ dise $\% \mathrm{C} 3 \% \mathrm{~B} 1 \mathrm{0} \% 20 \mathrm{de} \% 20 \mathrm{bombeo} . \mathrm{pdf}$

[4] P. A. W. Cornejo, "Diseño e implementación de plataforma scada para sistema de electrificación sustentable en la localidad de Huatacondo," Tesis de pregrado, Santiago de Chile: Universidad De Chile, 2011.

[5] G. F. I. Baldinia, "An early warning system for detecting GSM-R Wireless interference in the high-speed railway infrastructure." International journal of critical infraestructure protection, vol. 3, p.140-156, 2010. Doi: https://doi.org/10.1016/j. ijcip.2010.10.003.

[6] Alcaldía de Ocaña, "Plan Maestro Acueducto y Alcantarillado Ocaña," 1993. [Online]. Available: https://colaboracion.dnp.gov.co/CDT/Conpes/ Econ $\% \mathrm{C} 3 \% \mathrm{~B} 3 \mathrm{micos} / 2614$.pdf

[7] K. Ogata, Ingeniería de control moderna, Madrid: Pearson Educación, S.A., 2010.

[8] M. S. H. P. F. Jalilian, "Design and implementation of the monitoring and control systems for distribution transformer by using GSM network." Electrical Power and Energy Systems, vol. 76, p. 36-41, January 2016. Doi: https://doi.org/10.1016/j.ijepes.2015.07.022.

[9] R. A. García-León, E. Flórez Solano, and E. Pérez Rojas, "Diseño de una maquina amasadora y laminadora automática de masa para pan," Revista Ingenio, vol. 8, no. 1, pp. 59-71, 2015. Doi: https:// doi.org/10.22463/2011642X.2048

[10] R. A. García-León and S. Suarez Castrillón, "Diseño de un prototipo de sembradora mecánica de granos, alternativa agrícola," Revista Ingenio, vol. 12, no. 2011-642X, pp. 33-40, 2016. Doi: https://doi. org/10.22463/2011642X.2122

[11] Mateus Guerra, T. P. " Sistema de monitoreo y control remoto con acceso inalámbrico bidireccional a procesos industriales." Revista Colombiana de Tecnologías de Avanzada. , vol. 1, no.17, pp.56-61, 2011. Doi:10.24054/16927257.v12.n17.2011.171

[12] Ogata, K. Ingeniería de control moderna. Madrid: Pearson Educación, S.A, 2010

[13] A. M. Rodríguez-Suarez, J. A. Moreno-Montagut, \& M. Trigos-Rodríguez, "Los videos tutoriales como herramienta formativa", Revista Ingenio, vol. 10(1), pp. 37-42, jun. 2017. Doi: https://doi. org/10.22463/2011642X.2077

[14] C. M. Durán-Chinchilla, M. Cárdenas-García, \& T. Velásquez-Pérez, "Los modelos pedagógicos y su 
influencia en la práctica docente de la Universidad Francisco de Paula Santander", Revista Ingenio, vol. 9(1), pp. 77-88, jun. 2016. Doi: https://doi. org/10.22463/2011642X.2068

[15] R. A. García-León, Álvaro Avendaño-Quintero, \& S. A. Suarez-Castrillón, "Diseño de un prototipo de sembrador mecánica de granos, alternativa agrícola", Revista Ingenio, vol. 12(1), pp. 33-40, ene. 2017. Doi: https://doi.org/10.22463/2011642X.2122

[16] G. Guerrero-Gómez, E. Espinel-Blanco, \& T. Velásquez-Pérez, "Análisis isocinético y corrección a condiciones de referencia en horno a cielo abierto en el municipio de Ocaña, Norte de Santander", Revista Ingenio, vol. 14(1), pp. 43-51, jul. 2017. Doi: https:// doi.org/10.22463/2011642X.2194

[17] A. A. Rosado-Gómez, "Consolidación de indicadores institucionales utilizando bodega de datos", Revista Ingenio, vol. 11(1), pp. 53-63, dic. 2016. Doi: https:// doi.org/10.22463/2011642X.2094 